\title{
The relative contribution of scene context and target features to visual search in scenes
}

\author{
Monica S. Castelhano and Chelsea Heaven \\ Queen's University, Kingston, Ontario, Canada
}

\begin{abstract}
Many experiments have shown that knowing a target's visual features improves search performance over knowing the target name. Other experiments have shown that scene context can facilitate object search in natural scenes. In this study, we investigated how scene context and target features affect search performance. We examined two possible sources of information from scene context - the scene's gist and the visual details of the scene-and how they potentially interact with target-feature information. Prior to commencing search, participants were shown a scene and a target cue depicting either a picture or the category name (or no-information control). Using eye movement measures, we investigated how the target features and scene context influenced two components of search: early attentional guidance processes and later verification processes involved in the identification of the target. We found that both scene context and target features improved guidance, but that target features also improved speed of target recognition. Furthermore, we found that a scene's visual details played an important role in improving guidance, much more so than did the scene's gist alone.
\end{abstract}

When searching for items in our daily lives, we typically search for things that are familiar in places that are familiar, such as a knife in your kitchen. Inevitably, the search is much faster when you search for a knife in your own kitchen than in a friend's or a complete stranger's kitchen. But even in a stranger's kitchen, you would still know to look in drawers or on the countertop. Thus, the types of top-down influences arising from scene context in each of these circumstances can come from a multitude of sources. It can include general semantic knowledge (like in a stranger's kitchen) or knowledge of specific visual details or features (like in your own kitchen). The question remains whether either one or both of these top-down influences (general semantic and specific visual details) primarily affect how we search and whether target-object features may change this. In the present study, we examined the contributions made by top-down knowledge of scene context and target features to searching in scenes.

\section{Scene Context and Search Guidance}

From early on, scene context has been known to be an important factor in determining eye movement and attention allocation (Buswell, 1935; Yarbus, 1967). Since then, many other studies have shown an effect of scene context on visual search (Castelhano \& Henderson, 2007; Ehinger, Hidalgo-Sotelo, Torralba, \& Oliva, 2009; Neider \& Zelinsky, 2006; Torralba, Oliva, Castelhano, \& Henderson, 2006; Võ \& Schneider, 2010). Despite the many years of research, it is still unclear just how scene context affects visual search. There are many types of top-down influences derived from scene context discussed in the litera- ture, but these are often vague, fuzzy, or confounded with multiple sources of scene information. For example, scene context could affect search through semantic associations between the search target and overall scene (Friedman, 1979; Henderson, Pollatsek, \& Rayner, 1987; Hollingworth \& Henderson, 1998; Loftus \& Mackworth, 1978), on the basis of spatial layout and visual details of the scene alone (Brockmole \& Võ, 2009; Castelhano \& Henderson, 2007; Torralba et al., 2006), or through associations between other objects within the scene and the target object (Bar, 2004; Biederman, Mezzanotte, \& Rabinowitz, 1982; Davenport, 2007; Davenport \& Potter, 2004). In the present study, we examined two possible sources of information from scene context: general semantic information (or scene gist) and the visual details of the scene.

The influence of scene context on visual search has most often been attributed to general semantic knowledge about that scene's basic-level category (i.e., scene gist; Castelhano \& Henderson, 2007, 2008; Friedman, 1979; Henderson, Weeks, \& Hollingworth, 1999; Hock, Gordon, \& Whitehurst, 1974; Neider \& Zelinsky, 2006; Tatler, 2009). General semantic knowledge is thought to facilitate search by providing information about the target object's likelihood in a scene and its likely location (e.g., in kitchens, toasters are typically found on countertops and rugs on the ground) (Antes, 1974; Castelhano \& Henderson, 2007; Eckstein, Drescher, \& Shimozaki, 2006; Ehinger et al., 2009; Henderson et al., 1999; Neider \& Zelinsky, 2006; Torralba et al., 2006; Võ \& Schneider, 2010). Indeed, Henderson et al. (1999) found that consistent target objects (e.g., a toaster in a kitchen) were fixated 
much sooner than inconsistent target objects were (e.g., a toaster in a playground). They concluded that semantic information available for the consistent targets allowed participants to narrow down the possible locations and find those targets faster. More recently, Neider and Zelinsky (2006) showed that fixations were directed to the top half of a scene when searching for objects typically found in the sky (e.g., blimps, helicopters) and to the bottom half when searching for objects typically found on the ground (e.g., tanks, trucks). As in Henderson et al. (1999), when target objects were not placed in their typical locations in the scene, searches for these objects took much longer. In these cases, scene context had its influence based on the general locations where target objects were likely to be found.

In addition to a scene's semantic or categorical information, its visual details and spatial layout may have a strong influence on search. Scene context is operationalized as the learned association between the target placement and the global features of the scene (e.g., scale, layout, viewpoint) in the contextual guidance model (Ehinger et al., 2009; Torralba et al., 2006). When participants are searching for targets, the model matches the global scene features to where it has "learned" that the targets are most likely to occur. In a strict interpretation of the model, there is no need for semantic information; instead, it is the global features of a scene that are associated with the placement of the target object. Alternatively, a less strict interpretation would state that scene semantics are important in retrieving the relevant past experiences, but the visual details are necessary for determining the exact location to which the searcher should direct his or her attention (i.e., contextual priors). In either case, the visual details of the scene are essential in accounting for the effects of scene context.

There are also studies that suggest that visual details and spatial layout of a scene are equally, if not more, important than general semantic knowledge in driving visual search performance. Castelhano and Henderson (2007) had participants search for a target using a gazecontingent window, so that only foveated information was visible at any one time. When participants were shown a preview of the search scene before search, visual search performance was significantly improved. However, when a scene that was matched in category but not in visual details was shown, no improvement was found. Thus, although general semantic knowledge provides general location information, the scene's visual details may be more important in guiding eye movements and attention.

\section{Target Features}

Unlike with scene context, many studies have investigated how target knowledge affects search performance. Specifically, many studies have examined the relative contributions of the target's general category versus its visual features. For instance, using the most basic manipulation, studies have shown that search is far faster when the target is specified by an identical picture cue than by a name cue (Castelhano, Pollatsek, \& Cave, 2008; Foulsham \& Underwood, 2007; Malcolm \& Henderson, 2009; Vickery,
King, \& Jiang, 2005; Wolfe, Horowitz, Kenner, Hyle, \& Vasan, 2004; for a review, see Wolfe, 1998). In addition, Wolfe et al. found that, when the picture cue was a different exemplar of the target category, performance was no better than with a name cue. However, although search is facilitated by knowledge of the target's visual features, an exact match between cue and target image is not necessary for there to be some improvement in performance. Vickery et al. cued participants with an exact target image cue, a rotated image cue, or a name cue. They found that rotated image cues led to better performance than name cues did, although performance was not as good as when the cue was an exact image. Thus, even when the image cue did not depict the exact target orientation, search was more efficient, as long as some visual features were known. This was further demonstrated more recently by Schmidt and Zelinsky (2009), who found similar results: When participants were given a textual description of the target, more descriptive cues led to faster searches than did less descriptive ones.

\section{Combining Scene Context and Target Features}

When examining scene context and target features, one question that remains is whether target and scene information interact or affect search patterns independently. Ehinger et al. (2009) recently examined the contributions of both scene context and object features during visual search. The comparison was made using the contextual guidance model, in which information about scene context and information about target features were computed independently and were weighed equally in guiding visual search. When examining both scene context and target feature contributions to search performance, the model predicted fixated regions with high accuracy (89.5\%). Additionally, when each information source was assessed separately, scene context was a better predictor of fixation placement than was target feature information. However, the contribution of each source may depend on what is being searched and in what context: Ehinger et al. only examined visual search of people in city streets. In another recent study, Kanan, Tong, Zhang, and Cottrell (2009) proposed applying the saliency using natural statistics (SUN) model (Zhang, Tong, \& Cottrell, 2007; Zhang, Tong, Marks, Shan, \& Cottrell, 2008) to explain fixation placement during visual search. This model predicts fixation placement on the basis of target features alone. Kanan et al. found that, on the basis of target features alone, the SUN model could predict participants' eye movement patterns just as well as the original contextual guidance model, which is based entirely on scene context (Torralba et al., 2006). Thus, target features may account for search guidance in scenes just as well as scene context does. However, no study has informed participants of the exact target template to use for each search (as has been done in the target-feature studies discussed above). Therefore, it is difficult to know whether scene context and target features combine to aid search when both are known or whether they are, in fact, separate processes that affect search guidance independently. 


\section{The Present Study}

The present study investigates how scene context affects search and whether the top-down knowledge of target features and scene context combine. In two experiments, we controlled whether the visual features of the target and the visual details of the scene are known prior to search. Participants were shown either a picture of the scene they would be searching in or a word describing the scene gist (e.g., kitchen, living room, etc.). If the general semanticscene knowledge acquired from the name cue facilitates performance by helping viewers retrieve general global features about the scene and location information about the target, then, when such visual feature information is explicitly supplied from the picture cue, it should be redundant and not offer additional facilitation of performance over the scene-name cue. If the general semanticscene knowledge does not provide adequate information without the knowledge of the scene's visual detail, then better performance should result when the scene-picture cue is shown than when the name cue is shown.

In addition, participants were shown either an exact picture of the target or a word that described it. By manipulating availability of the scene's and the target's visual information, we were able to investigate the effectiveness of each type of top-down information on search performance and examine whether they would interact. If both the scene context and the target features contribute to search guidance independently, we would expect search performance to benefit from both. If these two sources of information interact, then a synergistic benefit to search performance may be evident, such that having both would be better than having either one individually. It is also possible that the presence of one source of information may decrease the effectiveness of the other. For instance, knowing the target's visual features may diminish the benefits to be derived from scene context if the search is guided by the target's visual features regardless of where they are in the scene.

\section{Components of Visual Search}

To more closely examine the effect of scene context and target features on eye movements during search, we looked at two processing components of search: attentional guidance and verification. Variations of these processes have been examined in past studies (Castelhano et al., 2008; Malcolm \& Henderson, 2009; Rutishauser \& Koch, 2007; Sheinberg \& Logothetis, 2001). First, attentional guidance is defined as the time from the onset of the scene until the first fixation on the target object. Previous studies have demonstrated that saccades tend to be directed toward items sharing target features (Findlay, 1997; Kanan et al., 2009; Scialfa \& Joffe, 1998; Williams \& Reingold, 2001). In addition, scene context may affect attentional guidance by limiting search to only target-relevant regions (Ehinger et al., 2009; Torralba et al., 2006). However, it is not yet clear whether scene context will be as effective when there is knowledge of target features or whether having both types of information may improve performance further.

Second, verification is defined as the time from the first fixation on the target object until a response is made. Consequently, this could include fixations on other objects, as well as fixations on the target, and, thus, would reflect the decision processes assessing the object's match to the target. McCarley, Kramer, Wickens, Vidoni, and Boot (2004) found that, with increased practice, the greatest improvement was in the recognition of the target once fixated, not in the likelihood of fixating it. More recently, Castelhano et al. (2008) found that picture cues, as compared with name cues, greatly reduced verification time and improved guidance to the target. It is not clear from other studies whether scene context (either the scene gist or visual details) would have a similar effect on target verification (Biederman et al., 1982; Davenport \& Potter, 2004; Hollingworth \& Henderson, 1998).

In Experiment 1, we investigated whether scene context and target feature information would interact across these two components. In Experiment 2, we further examined the contribution of the general semantic knowledge of scene context.

\section{EXPERIMENT 1}

\section{Method}

\section{Participants}

Thirty-two Queen's University undergraduate students participated for course credit or were compensated $\$ 10$ per hour.

\section{Stimuli and Apparatus}

Stimuli were 48 color photographs of indoor and outdoor scenes taken from various sources (e.g., Internet, catalogs, etc.). For each search scene, corresponding scene and target cues were created. The scene-picture cue was identical to the search scene, except that the target was digitally removed using Adobe Photoshop software ${ }^{1}$ (see Figure 1). The target-picture cue was the target copied from each search scene and pasted in the center of a gray background with no scene information included. Thus, all of the features of the target were identical between the scene and the cue, except for its location on the screen (it was always in the center for the target-picture cue). ${ }^{2}$ The scene-name and target-name cues comprised a single word in black text on a gray background describing the scene's gist or basiclevel category (i.e., kitchen, bathroom, etc.) and target basic-level category (i.e., toaster, lamp, etc.), respectively.

Eye movements were tracked using an EyeLink 2000 (SR Research), sampling eye movements at $1000 \mathrm{~Hz}$. The experimenter did not accept calibration unless the average spatial error was $0.40^{\circ}$ or less and the maximum error was no greater than $0.75^{\circ}$. Calibration checks between all trials ensured that the calibration error was kept low. Stimuli were displayed on a 21 -in. CRT monitor at a resolution of $800 \times 600$ pixels and a $100-\mathrm{Hz}$ refresh rate. Images subtended $38.1^{\circ} \times 28.6^{\circ}$ of visual angle, and targets subtended about $2.5^{\circ} \times 2.38^{\circ}$, on average.

\section{Procedure}

Participants were seated $60 \mathrm{~cm}$ away from the monitor on a headrest. Participants were instructed to look for a target in a photograph and were told they would receive a hint about the scene and/or target. When they found the object, they were instructed to look at it and push a button. Participants were then calibrated on the eyetracker using a nine-point calibration screen.

For each trial, the scene cue was shown for $1 \mathrm{sec}$, followed by a fixation cross for $500 \mathrm{msec}$, and then by the target cue for $1 \mathrm{sec}$ (see Figure 1A). The cues were followed by the words "Get ready!" at the center of the screen and then by the search scene. The target-picture and -name cues were always presented at the center of the screen. The search would end either when participants pressed a button or after $15 \mathrm{sec}$. The target was present in every search scene, but it was never present in the scene-picture cue. There were 48 experimental and 3 practice trials. Scenes were counterbalanced across the 


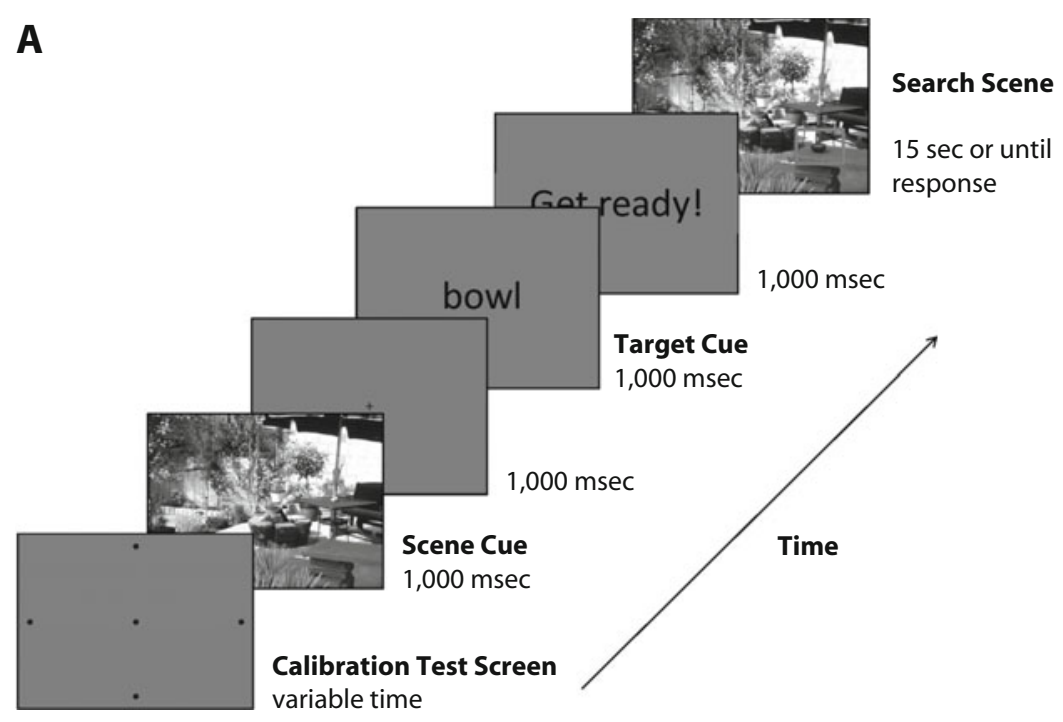

B

Word

Picture

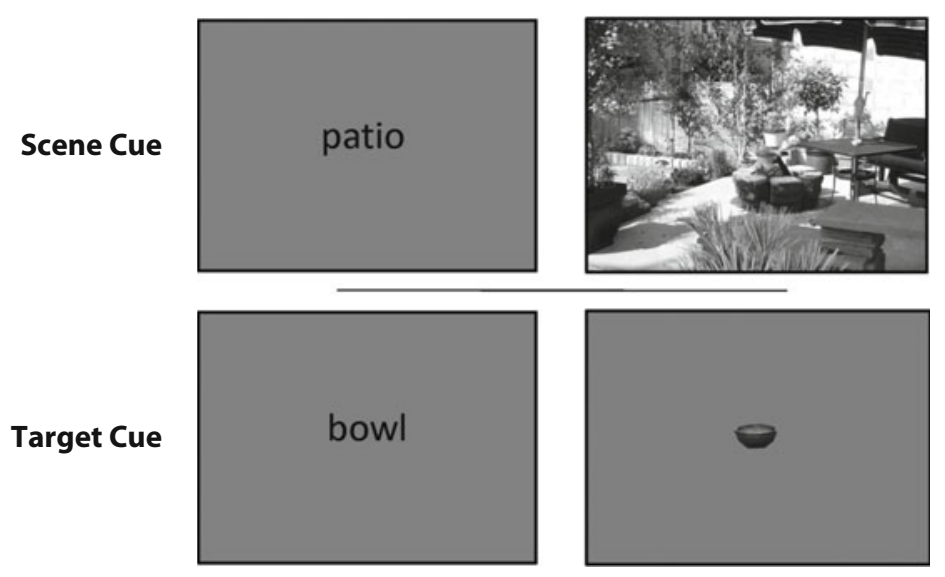

Figure 1. (A) Example screen shots of one trial for Experiments 1 and 2. This example shows the scene-picture cue and the target-name cue. (B) Example of cues for the scene and target in both the name and picture cue conditions in Experiment 1 . The scenes were presented in color.

two scene-cue (scene-picture and scene-name) and two target-cue (target-picture and target-name) conditions for each participant and were randomly presented. Thus, participants saw each search scene once, and the conditions were fully crossed. The experiment lasted approximately $30 \mathrm{~min}$.

\section{Results}

To investigate the relative effects of target feature and scene context information on visual search, we examined accuracy rates and reaction times (RTs) first; then we examined the global eye movement measures. Finally, we grouped further eye movement measures into the two search components: attentional guidance and verification. For all eye movement analyses, each target was defined by a rectangle surrounding it, approximately $1^{\circ}$ from its edge.

\section{Accuracy Rates}

Average accuracy across conditions is presented in Table 1. Incorrect trials occurred when either the target was not found within the 15 -sec search limit or the button was pressed before the target was fixated. Using a twoway, within-participants, 2 (target cue) $\times 2$ (scene cue) ANOVA, we examined the contribution of target features (target picture vs. target name) and scene-context information (scene picture vs. scene name). Overall, the accuracy rates were high $(85 \%)$, and there were no significant differences between the target-cue $[F(1,31)=1.17$, n.s.] and scene-cue $[F(1,31)=0.04$, n.s.] conditions and no interaction $[F(1,31)=0.2$, n.s. $]$. Only correct trials were included in the RT and eye movement analyses reported below. 
Table 1

Behavioral and Eye Movement Measure Means (and Standard Errors) $\times$ Scene-Cue and Target-Cue Conditions

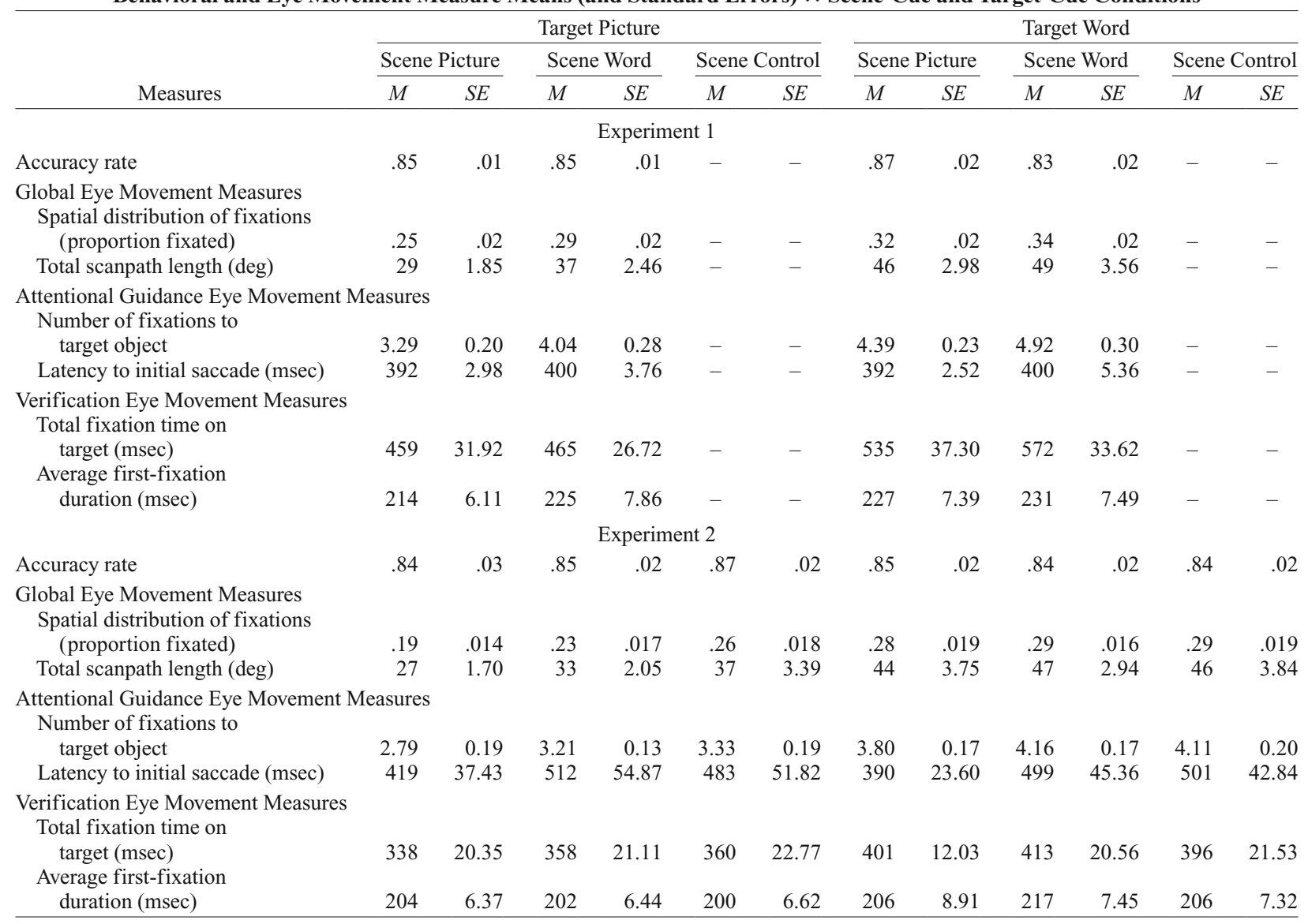

\section{RTs}

RTs were measured from the onset of the scene until the response button was pressed. On average, the participants took approximately $2 \mathrm{sec}$ to respond (see Figure $2 \mathrm{~A}$ ). We found that searches with a target-picture cue were executed significantly faster than were those with a target-name cue $[F(1,31)=28.9, p<.01]$, and searches with a scene-picture cue were executed significantly faster than were those with a scene-name cue $[F(1,31)=5.5$, $p<.05]$. The interaction between target cue and scene cue did not reach significance $[F(1,31)=0.06$, n.s.]. Thus, it seems that scene and target information may be additive. Although there is no strong case for anything other than additive effects, it should be noted that variability across participants was high. As a result, we cannot definitively conclude that no interaction exists.

\section{Global Eye Movement Measures}

Before examining each of the visual search component measures, we analyzed global measures, which include eye movements made across the entire trial. On the basis of findings from previous studies, information about the target and scene most likely affected how fixations were distributed across the scene. We therefore examined the spatial distribution of fixations and the total scanpath length.

Spatial distribution of fixations. Figure 3 depicts the spatial distribution of fixations for a scene for all participants in the same experimental conditions. The figure was created by weighting each fixation according to the fixation's duration and convolving a fixation map with a Gaussian filter. The Gaussian filter was defined by a standard deviation of $1^{\circ}$ in order to approximate the size of the fovea (for similar analyses, see Castelhano, Mack, \& Henderson, 2009; Henderson, 2003; Pomplun, Ritter, \& Velichkovsky, 1996; Zelinsky \& Schmidt, 2009). ${ }^{3}$ To quantify the spatial distribution of fixations, we computed the percentage foveated across participants for each scene. To calculate the foveated area, a circular filter $\left(1^{\circ}\right.$ radius $)$ was placed centered on each fixation for each scene under each viewing condition; thus, for each scene, the summed area occupied by the circular filters represented the total area fixated. Mean spatial distributions are presented in Table 1. We found that there was a significant effect of target cue $[F(1,47)=12.74, p<.01]$, where the picture showed significantly less dispersion than did the name, as well as a significant effect of scene cue, also showing that the picture led to less dispersion than did the name 

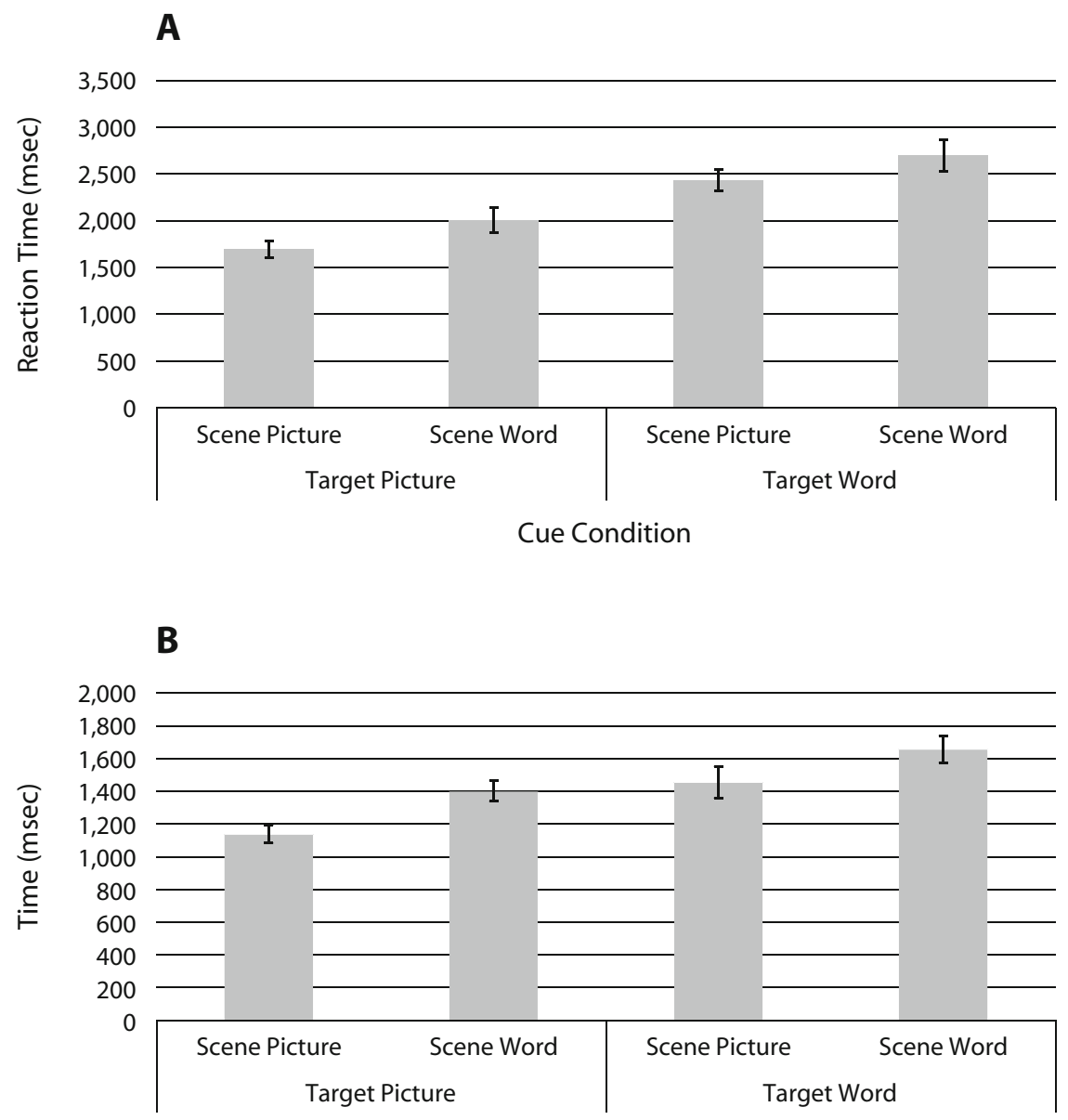

Cue Condition

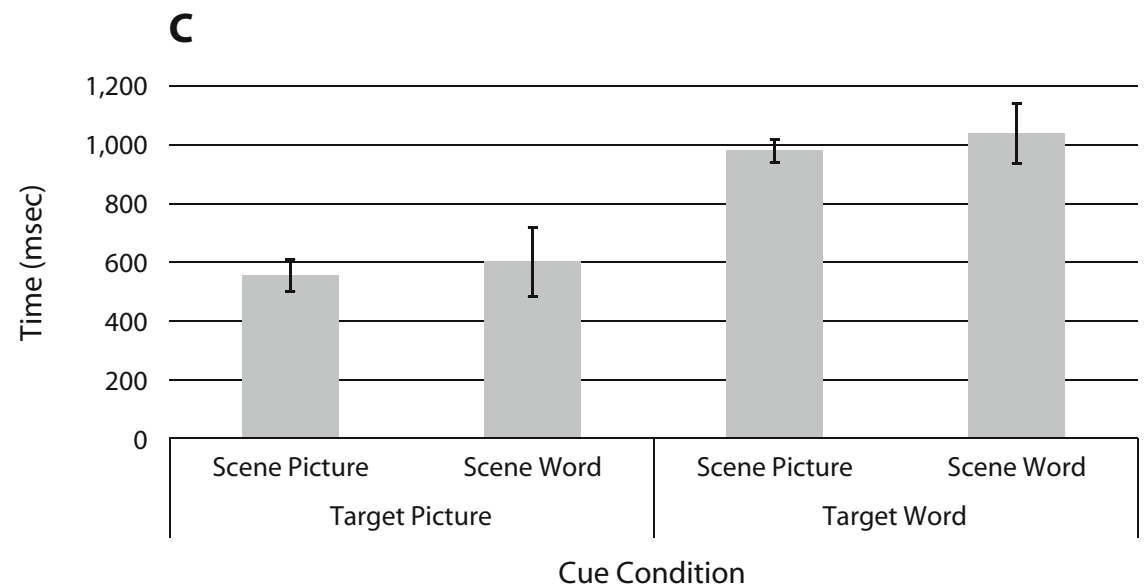

Figure 2. Means across the target- and scene-cue conditions in Experiment 1. Panel A shows reaction times, panel $B$ shows latency to the target object, and panel $C$ shows verification times of the target object. Error bars represent standard errors of the means.

$[F(1,47)=7.77, p<.01]$. However, there was no significant interaction $[F(1,47)=0.76$, n.s.].

Total scanpath length. As an additional measure of the dispersion of fixations in each of the cue conditions, we also calculated the total scanpath length. This measure is a sum of the lengths of all of the saccades and allows us to assess whether scanning was predominated by longer saccades in any one of the conditions (for a similar analysis, see Castelhano et al., 2009). In degrees of visual angle, we found the same pattern of results with shorter scanpath 


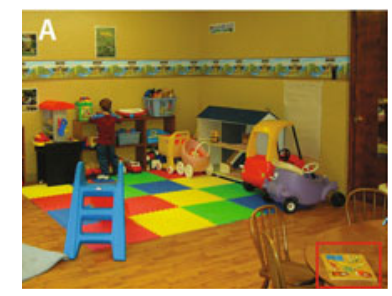

Scene-Picture Cue
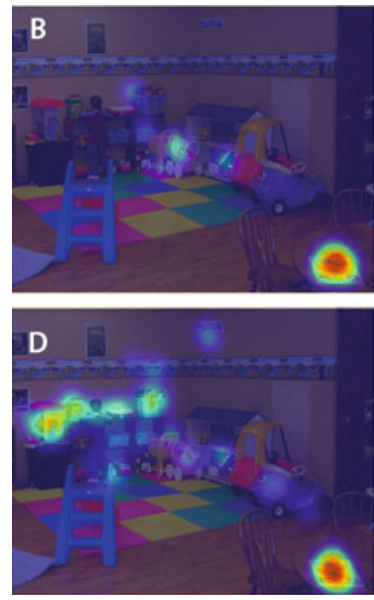

Scene-Name Cue
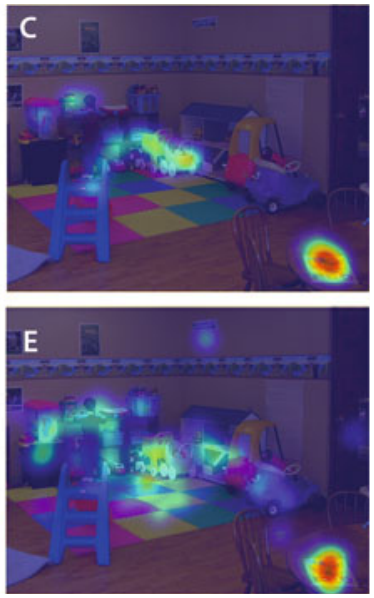

Figure 3. The distribution of all fixations for all participants over the same scene. Panel A shows the original image, in which the participants searched for a puzzle (highlighted by the red box). The images in the other panels show the placement of all fixations in the (B) target-picture/scene-picture, (C) target-picture/scene-name, (D) target-name/scene-picture, and (E) target-name/scene-name cue conditions. As can be seen qualitatively in this figure, fixations tended to be less distributed for each of the picture cues than for the name cues. For each condition, the proportions of scene fixated were $.20, .27, .43$, and .54 for the images in panels $B, C, D$, and $E$, respectively.

lengths for the target-picture cue $[F(1,31)=22.80, p<$ $.01]$ and shorter scanpath lengths for the scene-picture cue $[F(1,31)=5.25, p<.01]$, but no interaction $[F(1,31)=$ 1.06 , n.s.].

Attentional guidance. Of the two visual search components described above, we first examine the attentional guidance eye movement measures. Information about the target or scene that would affect the amount of time until the target was fixated, or the number of fixations needed to find the target, would be reflected in these measures.

Target latency. Target latency is perhaps the most basic measure of attentional guidance because it reflects the total amount of time spent during the initial search for the target, from the onset of the search scene until the first fixation on the target (excluding the first fixation). The mean target latencies for each condition are presented in Figure 2B. As with RT, we found that both target-picture and scene-picture cues led to shorter latencies than their respective name cues did [for target cues, $F(1,31)=$ $21.68, p<.01$; for scene cues, $F(1,31)=9.51, p<.01]$. Again, there was no significant interaction between target and scene cues $[F(1,31)=0.20$, n.s. $]$.

Number of fixations to target. Another measure of the efficiency of the initial search for the target is the num- bers of fixations made. The mean numbers of fixations for each condition are presented in Table 1. As may be apparent, this measure refers to the number of fixations from the scene onset until the first fixation on the target (excluding the first fixation). Although related to target latency, this measure reveals whether participants are effectively selecting likely target candidates for fixation. We found that participants made about one fewer fixations with the target-picture cue $[F(1,31)=29.04, p<.01]$ than with the target-name cue and about one fewer fixations with the scene-picture cue $[F(1,31)=4.95, p<.01]$ than with the scene-name cue. As was found for the measures above, there was no significant interaction between scene cue and target cue $[F(1,31)=0.20$, n.s.].

Initial saccade latency. It could be hypothesized that the advantage of being shown a scene-picture cue occurs within the first few fixations. Because scene gist is acquired from the first glance at a scene, those who had the word cue would get the scene gist very soon after the scene onset. Thus, the advantage for those who had a picture cue would be while this initial processing takes place. The initial saccade latency is a measure of the amount of time until the execution of the first saccade after the search scene onset. We found that the scene-picture cue 
led to significantly shorter latencies than did the scenename cue $[F(1,31)=8.3, p<.01]$, but there was no effect of target cue $[F(1,31)=0.01$, n.s. $]$ and no interaction $[F(1,31)=0.02$, n.s. $]$.

Verification. We also examined the verification time measures, which are included in the time from the first fixation on the target until the button is pressed. These measures could reflect fixations on the target or fixations on other objects before refixating the target and would therefore reflect how quickly the target was identified as such and how certain the participant was about the target.

Verification time. As a basic measure, we examined the time lapsed from the start of the first fixation on the target until the response. As mentioned above, this measure includes time spent on the target and on other objects after it was fixated. We found that the target-picture cue led to shorter verification times than did the target-name cue $[F(1,31)=17.4, p<.01]$. However, unlike the outcome of the analyses for the attentional guidance measure, there was no effect of scene cue $[F(1,31)=0.30$, n.s.]. So, although the scene information seemed to have an effect on the guidance to the target, once it was fixated, knowing the scene's visual details had no effect. As with the attentional guidance measures and RT, there was no significant interaction $[F(1,31)=0.09$, n.s. (see Figure $2 \mathrm{C}$ ).

Total time on target. To examine the total time spent processing that target, we measured the total time spent viewing the target before the participants pressed the button. The total time was computed as the sum of all of the fixations made on the target. We found that the total time on the target was less for the target-picture cue than for the target-name cue $[F(1,31)=27.74, p<.01]$, but there was no effect of scene cue $[F(1,31)=1.96$, n.s. $]$ and no interaction $[F(1,31)=0.06$, n.s.].

First-fixation duration on target. The first-fixation duration on an object has often been taken to indicate the initial recognition of the object at fixation (Henderson, 1992; Rayner \& Pollatsek, 1992). Here, we measured it to see whether the target cue would have an immediate effect on the examination of the object or whether it may occur only in subsequent fixations (de Graef, Christiaens, $\&$ d'Ydewalle, 1990). We found that the first-fixation durations were marginally shorter for target-picture conditions than for target-name conditions $[F(1,31)=3.58$, $p=.068]$. As was true for the measures above, there was no effect of scene $[F(1,31)=1.57$, n.s. $]$ and no interaction $[F(1,31)=0.30$, n.s. $]$

\section{Discussion}

The results of this first experiment indicate that both scene context and target features contribute to search performance overall. When we examined the two components of search through the eye movement measures, we found that both scene and target information contribute to the attentional guidance, and the results suggest that they do so additively. We also found that target feature information further decreased search times by decreasing the time to identify the target object once it was fixated. Yet, the help with the identification of the target was not immediately apparent, as indicated by the first-fixation duration measure. The strong effect of target cue on the total time spent examining the target suggests that this information had an effect on the durations of subsequent fixations on the target.

It is important to note that, from the results of the first experiment, it is impossible to say how much guidance is obtained from the conceptual scene information alone (i.e., scene-name cue). As discussed in the introduction, it may be that having scene-context information from only the scene's gist might provide some benefit (Castelhano \& Henderson, 2007; Friedman, 1979; Henderson et al., 1999; Neider \& Zelinsky, 2006; Tatler, 2009; Torralba et al., 2006). In order to address this question, a second experiment was conducted, in which a no-scene-cue control condition was added.

\section{EXPERIMENT 2}

In Experiment 2, we wanted to more closely examine how scene context affects search performance. We used the same experimental setup as in Experiment 1, with an added control condition with no scene information (i.e., the no-scene-cue condition). By comparing performance in the other scene conditions with the scene-control condition, we can investigate how general information based on the scene's gist affects both the search overall and the two processing components: attentional guidance and verification.

\section{Method}

\section{Participants}

Thirty Queen's University undergraduate students participated and were compensated $\$ 10$ per hour. None of the participants in Experiment 2 had participated in Experiment 1.

\section{Stimuli and Apparatus}

The stimuli and apparatus were identical to those in the first experiment, with one exception. A scene-control cue was added, in which no information about the scene was shown, and participants saw only a row of dashes presented on a gray background (see Figure 4). Thus, there were three scene-cue conditions (i.e., scenepicture, scene-name, scene-control), in addition to the two target-cue conditions, for six conditions in all.

\section{Procedure}

The procedure was the same as that in Experiment 1.

\section{Results}

As in Experiment 1, we examined accuracy rates, RTs, and eye movement measures. In addition, the eye movement measures were again grouped into two search components: attentional guidance and verification. For the eye movement measures, the same target definitions as in Experiment 1 were used.

\section{Accuracy Rates}

Correct responses were calculated in the same way as in Experiment 1: Incorrect trials were counted either when the target was not found within the 15 -sec search limit or when the button was pressed before the target was 


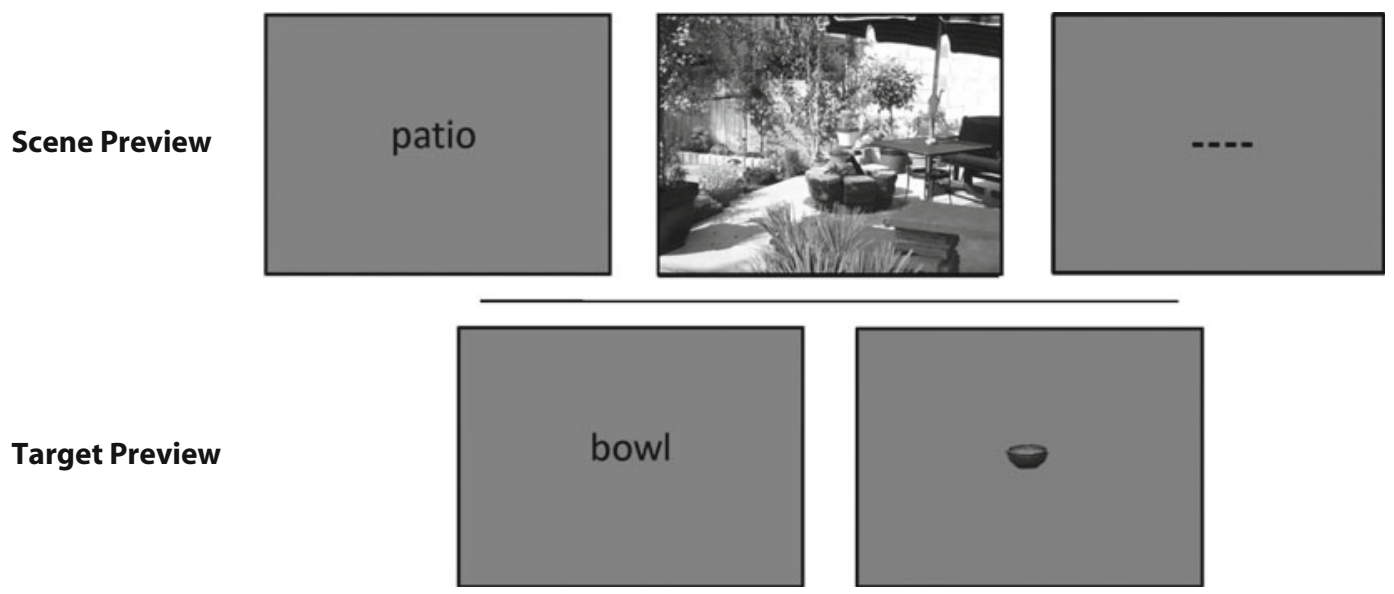

Figure 4. Example of cue conditions for the scene cue (name, picture, control) and target cue (name, picture) in Experiment 2.

fixated. Using a two-way, within-participants, 2 (target cue) $\times 3$ (scene cue) ANOVA, we examined the contribution of target features (target picture, target name) and scene-context information (scene picture, name, control). Overall, the accuracy rates were high and were equal to those found in Experiment 1 (85\%). Accuracy rate means are presented in Table 1. There were no significant differences between target-cue conditions $[F(1,29)=0.18$, n.s.] or among scene-cue conditions $[F(2,58)=0.26$, n.s. $]$ and no interaction $[F(2,58)=0.70$, n.s.]. Only correct trials were included in the RT and eye movement analyses reported below.

\section{RTs}

As in the previous experiment, RT was measured from the onset of the scene until the response button was pressed. Means are shown in Figure 5A. As in Experiment 1, participants took about $2 \mathrm{sec}$ to complete the search. We found that the target-picture cue led to faster searches than did the target-name cue $[F(1,29)=105.7$, $p<.01]$. In addition, there was a main effect of the scene cue $[F(2,58)=4.7, p<.05]$, and further planned comparisons showed that the scene-picture cue was faster than both the scene-name $[t(29)=2.7, p<.05]$ and the scenecontrol $[t(29)=2.4, p<.05]$ cue conditions. Interestingly and somewhat surprisingly, the difference between the scene-name and scene-control cues was nonsignificant $[t(29)=0.24$, n.s. $]$. Replicating the results of Experiment 1 , there was no interaction between target cues and scene cues $[F(2,58)=0.09$, n.s.].

\section{Global Eye Movement Measures}

As in Experiment 1, before examining each of the visual search component measures, we examined two global eye movement measures: spatial distribution of fixations and total scanpath length.

Spatial distribution of fixations. Figure 6 depicts the spatial distribution of fixations for a scene for all participants in each experimental condition. The figure was created using the same method described in Experiment 1.
Again, to quantify the spatial distribution of fixations, we computed the percentage foveated across participants for each scene; these values are presented in Table 1. Just as was found in other measures and in Experiment 1, we found a significant effect of target cue $[F(1,47)=28.37$, $p<.01]$, with the target-picture condition resulting in significantly less dispersion than the name condition, as well as a significant effect of scene cue, also showing that the picture led to less dispersion than the name did $[F(2,94)=5.35, p<.01]$. Interestingly, unlike in Experiment 1 , there was also a marginally significant interaction $[F(2,94)=2.98, p=.056]$. Further analyses using Tukey's LSD revealed that, when the target name was shown, the scene-cue factor had no effect; however, when the target picture was shown, fixations were significantly less dispersed in the scene-picture condition than in the scene-name $(p<.05)$ and scene-control $(p<.001)$ conditions. There was a marginal difference in dispersion between the scene-name and scene-control conditions $(p<.082)$, so it seems that the marginally significant interaction was caused by differences between the scene cues when the target-name cue (versus the target-picture cue) was shown. When we performed the same analyses excluding the control scene-cue condition (i.e., no cue), we saw the same pattern of results as in Experiment 1: For target cue, $F(1,47)=33.72, p<$ .01 ; for scene cue, $F(2,94)=4.81, p<.01$; and for the interaction, $F(2,94)=0.96$, n.s. This marginal interaction will be addressed further in the Discussion.

Total scanpath length. As in Experiment 1, we calculated the total scanpath length as an additional measure of the dispersion of fixations in each of the target- and scenecue conditions. As was mentioned above, this measure is a sum of the lengths of all of the saccades and allows us to determine the scanning pattern implemented, as measured by saccade lengths. We found that the target-picture cue produced significantly shorter scanpath lengths than did the target-name cue $[F(1,31)=29.97, p<.01]$. In addition, there was a marginal effect of scene cue $[F(2,62)=$ $2.44, p=.096]$. Further analyses revealed significantly 
A

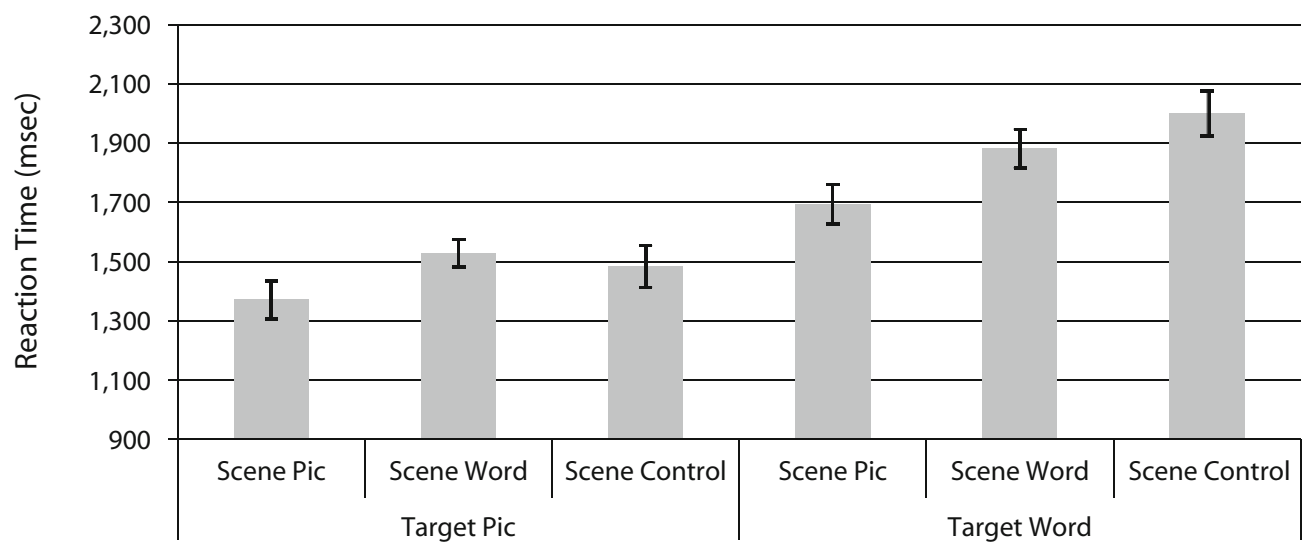

Cue Condition

B

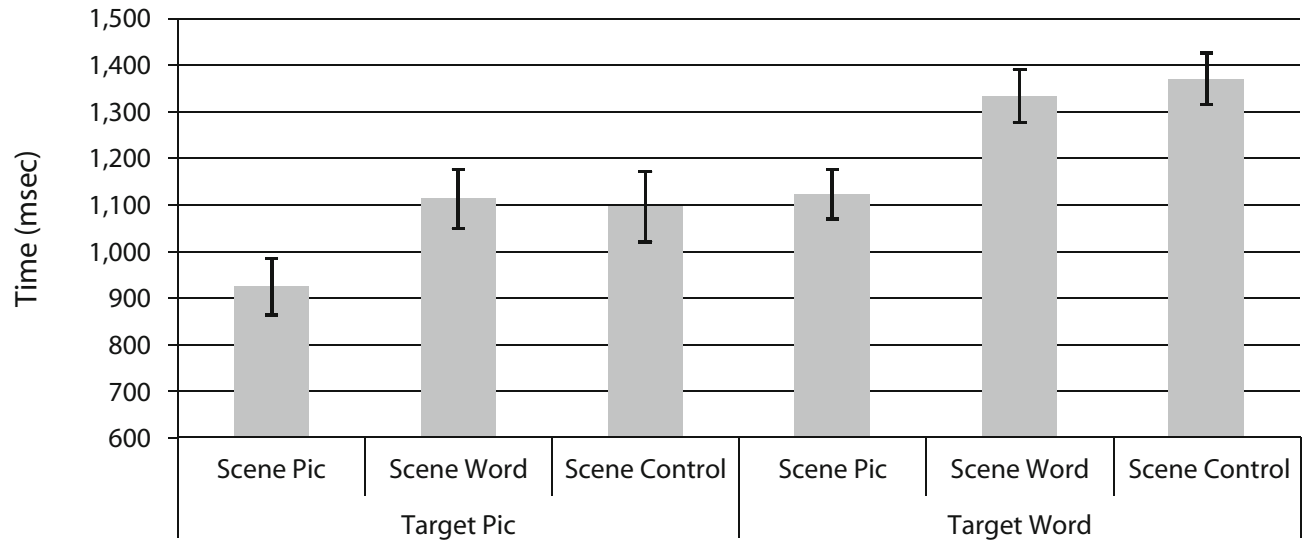

Cue Condition

C

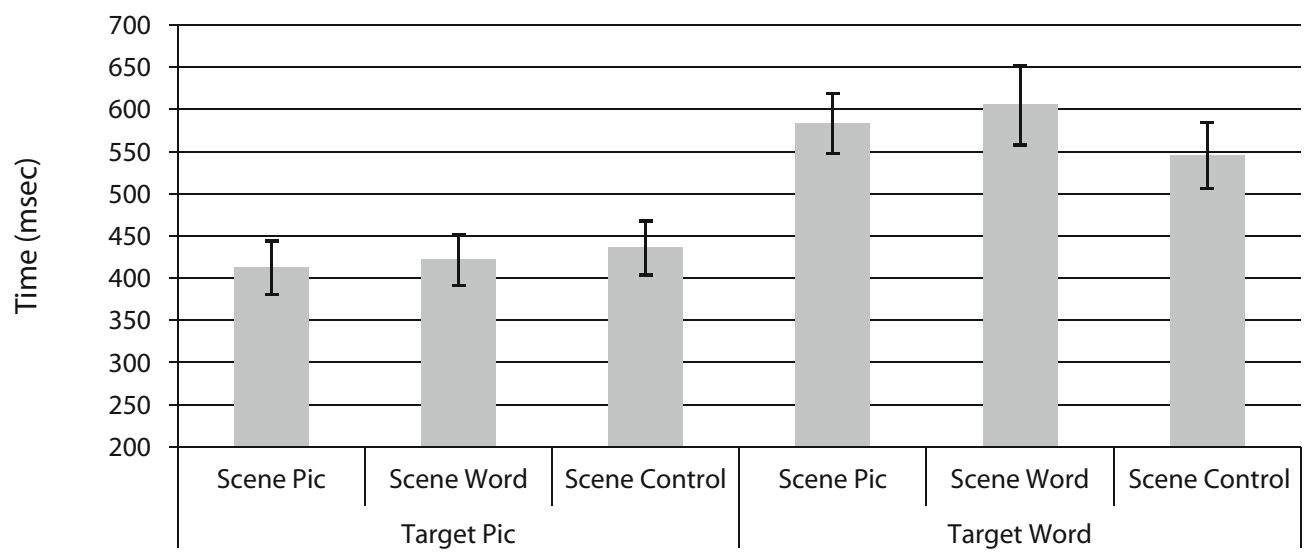

Cue Condition

Figure 5. Means across the target- and scene-cue conditions in Experiment 2. Panel A shows reaction times, panel $B$ shows latency to the target object, and panel $C$ shows verification times of the target object. Error bars represent standard errors of the means. 


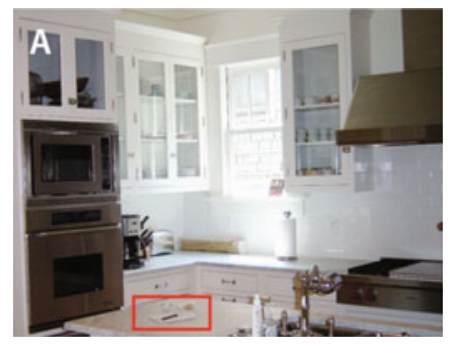

Scene-Picture Cue
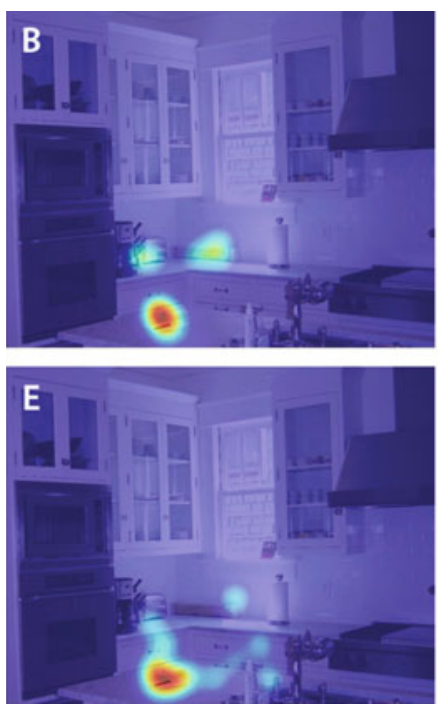

Scene-Name Cue
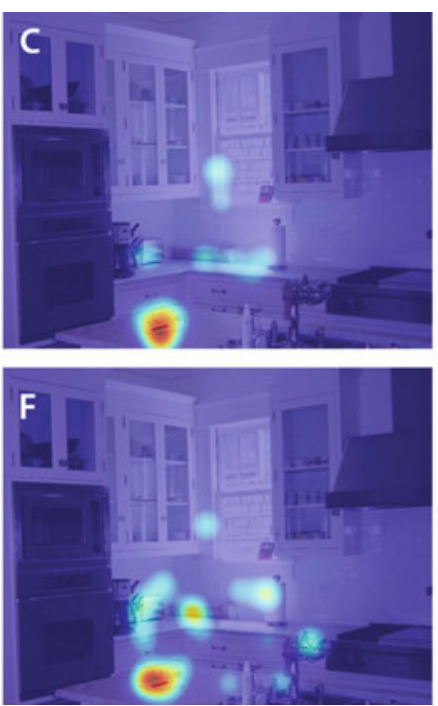

Scene-Control Cue
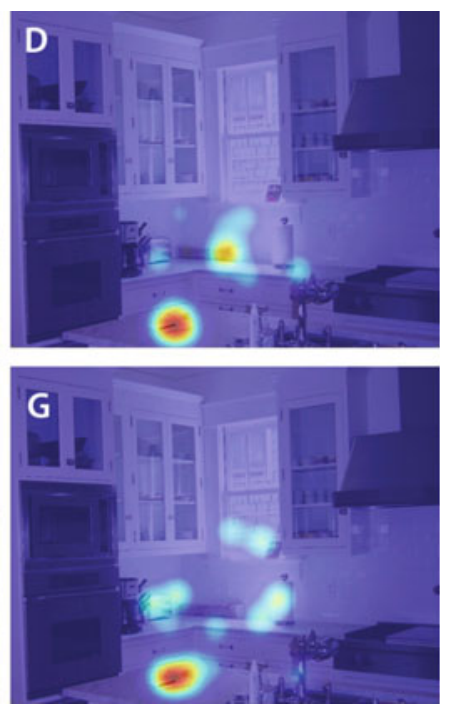

Figure 6. The distribution of all fixations for all participants over the same scene. Panel A shows the original image, in which participants searched for a notepad (highlighted by the red box). The images in the other panels show the placement of all fixations in the (B) target-picture/scene-picture, (C) target-picture/scene-name, (D) target-picture/scene-control, (E) target-name/scene-picture, (F) target-name/scene-name, and (G) target-name/scene-control cue conditions. As can be seen qualitatively across the images, fixations tended to be less dispersed for the picture cues than for the name and control cues. For each condition, the proportions of scene fixated were $.14, .24, .22, .11, .18$, and .17 for the images in panels $B, C, D, E, F$, and $G$, respectively.

shorter scanpath length for the scene-picture cue than for the scene-control cue and a marginal difference between the scene-picture and scene-name cues $(p=.063)$. The interaction was not significant $[F(2,62)=0.82$, n.s. $]$.

Attentional guidance. As in Experiment 1, we examined the attentional guidance eye movement measures first. These once more included target latency, number of fixations to the target, and initial saccade latency.

Target latency. Mean latencies are presented in Figure 5B. The target latency was again defined as the duration from the onset of the search scene until the first fixation on the target (excluding this first fixation). As with RT, we found that target-picture cues led to shorter latencies than did the target-name cues $[F(1,29)=19.4, p<$ $.01]$, and we found a main effect of scene cue $[F(2,58)=$ $7.71, p<.05]$. Planned comparisons among scene-cue conditions showed that the scene-picture cue led to faster searches than did the scene-name $[t(29)=3.3, p<.05]$ and scene-control $[t(29)=3.2, p<.05]$ cues. As was found for RTs, there was no significant difference between the scene-name and scene-control $[t(29)=0.20$, n.s.] cues. In addition, we found no significant interaction between target and scene cues $[F(2,58)=0.7$, n.s. $]$.

Number of fixations to target. The number of fixations to the target was measured from the search scene onset until the first fixation on the target (excluding this first fixation). The mean numbers of fixations across targetand scene-cue conditions are presented in Table 1. The analysis shows that participants made about one fewer fixations with the target-picture cue $[F(1,29)=32.29$, $p<.01]$ than with the target-name cue. There was also a main effect of scene cue $[F(2,58)=3.7, p<.01]$. Further analyses revealed that fewer fixations were made in the scene-picture-cue condition than in either the scene-name $[t(29)=2.47, p<.05]$ or the scene-control $[t(29)=2.25$, $p<.05]$ conditions. However, the scene-name and scenecontrol conditions did not differ significantly $[t(29)=$ 0.2 , n.s.]. As was found for target latency and in Experiment 1 , there was no significant interaction $[F(2,58)=$ 0.9 , n.s.].

Initial saccade latency. We again looked at the savings that the scene cue could provide in the initial search guid- 
ance. The initial saccade latency means are presented in Table 1. As was found in Experiment 1, there was a main effect of scene cue $[F(2,58)=5.47, p<.01]$, no effect of target cue $[F(1,29)=0.02$, n.s.], and no interaction $[F(2,58)=0.62$, n.s. $]$. Further analyses of the scene conditions showed that the scene-picture cue led to significantly shorter latencies than did the scene-name $[t(29)=$ $2.7, p<.05]$ and scene-control $[t(29)=2.5, p<.05]$ conditions. In addition, there was no difference between the scene-name and scene-control $[t(29)=0.6$, n.s. $]$ cues.

Verification. We also looked at the verification eye movement measures - that is, the time from the first fixation on the target until the button is pressed - to examine how quickly the target was identified after it was first fixated.

Verification time. This measure was defined as the time from the start of the first fixation on the target until the response. We found that the target-picture cue led to shorter verification times than the target-name cue $\operatorname{did}[F(1,29)=$ $50.27, p<.01]$. In addition, there was no effect of scene cue $[F(2,58)=0.33$, n.s. $]$ and no significant interaction $[F(2,58)=0.94$, n.s. $]$. Thus, as was found in Experiment 1 , there was a strong effect of the target cue, but no effect of the scene cue and no interaction (see Figure 5C).

Total time on target. When we look at the total time on the target object, we find the same pattern as with verification time. As before, the total time was computed as the sum of all of the fixations made on the target. The mean total times are presented in Table 1. We found that the total time on target was less for the target-picture cue than for the target-name cue $[F(1,29)=36.88, p<.01]$, and, as in Experiment 1, there was no effect of scene cue $[F(2,58)=$ 1.2, n.s.] and no interaction $[F(2,58)=0.83$, n.s. $]$.

First-fixation duration on target. We measured the first-fixation duration to again investigate whether target cue had an immediate effect on the examination of the target. As was done previously, the measure was defined as duration of the first fixation within the target region. The means are presented in Table 1. As was found in Experiment 1 , there was no effect of scene $[F(2,58)=0.63$, n.s.] and no interaction $[F(2,58)=0.50$, n.s.]. However, unlike in Experiment 1, where there was a marginal effect of the target cue, we found no effect of the target cue on the first-fixation duration $[F(1,29)=1.95$, n.s. $]$.

\section{Discussion}

From the second experiment, we can make two interesting conclusions. First, in comparison with the no-scene control, there was no effect of scene gist (available from the scene name). Participants did not perform better in the scene-name condition than in the scene-control condition. This was unexpected, because we had hypothesized that, even if search guidance was not specific, the general knowledge of the association between objects and scene category would guide attention. Instead, it seems that the visual details of the scene play an important role in guiding attention. We discuss this further in the General Discussion.
Second, this experiment demonstrated that, across the two component measures, there was no interaction between target features and scene context. However, we did find a marginally significant interaction for one of the global measures - namely, the spatial distribution of fixations. From this measure, it seems that, when the target picture was shown, there was less dispersion of fixations for the scene-picture condition than for the scene-control condition and marginally less dispersion of fixations for the scene-name condition than for the scene-control condition. One could hypothesize that, when given the target's visual detail, participants are better able to restrict their eye movements to relevant areas. That is, the effect of scene context may be easier to implement when specific knowledge about the target that could help identify its location in the scene (e.g., lighting information, viewpoint, scale, etc.) can be extracted. However, it is difficult to draw any strong conclusions from this result (especially since this pattern was not replicated in other measures), so we conclude that the two types of top-down information are additive.

\section{GENERAL DISCUSSION}

In the present study, we examined the effect of two types of top-down knowledge on search: target features and scene context. Across two experiments, two questions were addressed: (1) How does scene context contribute to search performance? and (2) Does scene-context information interact with target-feature information during search? To answer these questions, we manipulated the availability of the visual features of the target and of those of the scene before the search commenced. For the first experiment, participants were shown either a picture or name cue of the scene and either a picture or name cue of the target. In line with earlier studies (Castelhano et al., 2008; Malcolm \& Henderson, 2009; Vickery et al., 2005; Wolfe et al., 2004), RTs were much faster with target-picture cues than with target-name cues. Results also showed that a scene-picture cue improved search performance over the scene-name cue. We concluded that having the scene's visual details aided search more than did knowing general semantic information about the scene. In addition, we found that, across all measures, there was no interaction between the two sources of top-down information, so we concluded that they are additive and do not interact. In the second experiment, we further explored the contribution of scene context by adding a scene-control condition (with no scene information). Surprisingly, there was no difference between the scene-name and scene-control conditions, suggesting that visual details of the scene play an important role in speeding up search performance, but that general scenegist information does not.

One of the most unexpected findings was that scene context and target features did not interact. It seems that scene context did not limit target-feature search, and target features did not limit the influence of scene context on guidance. Of course, there could be a number of reasons 
for this. One possibility is that the system considers both types of information independently when selecting which location (on the basis of layout or of features detected in the periphery) has a higher likelihood of containing the target object. Another possibility is that, when both types of information are available, greater specificities of probable locations are selected for further scrutiny. Whatever the cause, the results highlight the fact that having both types of information improves performance more than either one alone. If one were to consider the relative contributions, it seems that, in the case of the scenes and targets used in the present study, target features seem to increase the effectiveness of search guidance more than scene context does. One way to think about this is to assume that, when the target features are known, those features can drive guidance more effectively to likely target candidates. However, it is unlikely that this will always be the case. As was mentioned above, Ehinger et al. (2009) found that scene context could account for fixation placement more than could the target features when searching for people in city streets. Thus, it is likely that different sets of scene and target combinations could result in various patterns.

We were also interested in whether target features and scene context affected attentional guidance and verification processes of visual search differently. In both experiments and across all attentional guidance eye movement measures, we found that knowing both the target features and scene context improved guidance and did so additively. Furthermore, from the results of Experiment 2, the benefit of scene context seems to arise from knowing the specific visual details of the scene in addition to the scene gist, but that general scene-gist information alone does not improve guidance during search. We discuss this surprising finding further below.

When we examined the effects of target features and scene context on verification times, a different pattern of results emerged. Although knowing the target's visual features improved search performance by shortening verification times, scene context had no effect. Our results support previous findings that show that the exact target features greatly decreased verification decision processes (Castelhano et al., 2008; Malcolm \& Henderson, 2009; McCarley et al., 2004). It should be noted, however, that our results do not speak directly to the debate about the effects of scene context on object recognition (Biederman et al., 1982; Davenport \& Potter, 2004; Hollingworth \& Henderson, 1998), because no manipulation of object consistency was included. However, this would be an interesting question to address in future studies on the components of visual search.

Interestingly, the target-picture cue had a significant effect on all verification measures with one exception: firstfixation duration. Because there was an effect of target feature on total time on target, it seems that the improvement of target verification in both experiments did not occur within the first fixation. Instead, subsequent examination of the target was shortened. Thus, target identification was faster with the target-picture cue, but the effect of this information was not immediate.
In Experiment 2, we were surprised to find that knowing the scene gist alone did not improve search performance. This finding suggests that the general knowledge about object placement in scenes may be driven by more specific visual information. That is, if you are looking for a toaster in a kitchen, you must know the general layout of the scene to know where the counter is, what angle you are looking at it from, and so forth, in order to effectively use the general semantic knowledge we have about a toaster's placement to guide eye movements. This is in contrast to the way most researchers characterize the effects of scene context on attention (Castelhano \& Henderson, 2007; Friedman, 1979; Henderson et al., 1999; Neider \& Zelinsky, 2006; Tatler, 2009). For instance, in Friedman's classic study, the main emphasis is on the scene schema and how well a particular object fits within it. The viewing times bore out this relationship, with very strange objects receiving much longer looking times than the more familiar objects did. In more recent studies, relations between objects and the scene have been operationalized either as correlates between the scene's global features and target placement (Ehinger et al., 2009; Torralba et al., 2006) or as target-feature similarity through the scene (Kanan et al., 2009). Thus, it would seem that, in order to demonstrate the advantage of scene context in search, semantic information about the scene gist might not be as essential as scene layout or visual features and their association with the target.

Alternatively, the effects of scene gist would only be seen within the first few milliseconds, since, once the search scene is shown, semantic and visual information can be extracted before the first saccade is executed. However, we should note that, although scene-picture cues did reduce the time until the first saccade was executed, the difference was only on the order of about $8 \mathrm{msec}$. Thus, it may not be that scene visual details led to faster initiation of the search but, rather, to improved planning. A scene's visual details could have led to participants' being better able to rule out certain areas unlikely to contain the target and have led, therefore, to fewer fixations and less time spent in the initial component of visual search: attentional guidance. Future studies are needed in order to address these two possible explanations for the lack of scene-gist effect on search guidance.

From the evidence presented here, we conclude that both scene context and target features have an effect on attentional guidance and that these effects are additive. The results also revealed that scene-context effects seem to be largely dependent on the specific visual details and spatial layout of a scene. In addition, knowing the target features further improves search performance by reducing verification time. Thus, theoretical accounts of visual search in real-world scenes must take into account the influences of both the scene information and the target information on overall search performance, as well as how each type of information affects component-search processes in turn.

\section{AUTHOR NOTE}

The authors thank Catherine Seohyun Jee for her help with data collection, as well as Ingrid Johnsrude, Sandy Pollatsek, Krista Ehinger, 
Carrick Williams, Mareike Wieth, Kristin Wiengartner, and Sian Beilock for helpful discussions and comments on earlier versions of the manuscript. The present research was supported by a Natural Science and Engineering Research Council of Canada grant, the Canada Foundation for Innovation, the Ontario Ministry of Research and Innovation, and an Advisory Research Committee grant to M.S.C. Correspondence concerning this article should be addressed to M. S. Castelhano, Department of Psychology, Queen's University, 62 Arch St., Kingston, ON, K7L 3N6 Canada (e-mail: monica.castelhano@, queensu.ca)

\section{REFERENCES}

ANTES, J. R. (1974). The time course of picture viewing. Journal of Experimental Psychology, 103, 62-70. doi:10.1037/h0036799

BAR, M. (2004). Visual objects in context. Nature Reviews Neuroscience, 5, 617-629.

Biederman, I., Mezzanotte, R. J., \& Rabinowitz, J. C. (1982). Scene perception: Detecting and judging objects undergoing relational violations. Cognitive Psychology, 14, 143-177. doi:10.1037/0278 -7393.9.3.411

Brockmole, J. R., \& Võ, M. L.-H. (2009, November). The role of semantic memory in learning contextual regularities in real-world scenes. Paper presented at the 50th Annual Meeting of the Psychonomic Society, Boston, MA.

Buswell, G. T. (1935). How people look at pictures: A study of the psychology of perception in art. Chicago: University of Chicago Press.

Castelhano, M. S., \& Henderson, J. M. (2007). Initial scene representations facilitate eye movement guidance in visual search. Journal of Experimental Psychology: Human Perception \& Performance, 33, 753-763. doi:10.1037/0096-1523.33.4.753

Castelhano, M. S., \& Henderson, J. M. (2008). The influence of color on the perception of scene gist. Journal of Experimental Psychology: Human Perception \& Performance, 34, 660-675. doi:10.1037/0096 $-1523.34 .3 .660$

Castelhano, M. S., Mack, M. L., \& Henderson, J. M. (2009). Viewing task influences eye movement control during active scene perception. Journal of Vision, 9(3, Art. 6). doi:10.1167/9.3.6

Castelhano, M. S., Pollatsek, A., \& Cave, K. R. (2008). Typicality aids search for an unspecified target, but only in identification and not in attentional guidance. Psychonomic Bulletin \& Review, 15, 795-801. doi:10.3758/PBR.15.4.795

DAVENPORT, J. L. (2007). Consistency effects between objects in scenes. Memory \& Cognition, 35, 393-401.

Davenport, J. L., \& Potter, M. C. (2004). Scene consistency in object and background perception. Psychological Science, 15, 559-564. doi:10.1111/j.0956-7976.2004.00719.x

de Graef, P., Christiaens, D., \& D'Ydewalle, G. (1990). Perceptual effects of scene context on object identification. Psychological Research, 52, 317-329. doi:10.1007/BF00868064

Eckstein, M. P., Drescher, B. A., \& Shimozaki, S. S. (2006). Attentional cues in real scenes, saccadic targeting, and Bayesian priors. Psychological Science, 17, 973-980. doi:10.1111/j.1467 $-9280.2006 .01815 . x$

Ehinger, K. A., Hidalgo-Sotelo, B., Torralba, A., \& Oliva, A. (2009). Modelling search for people in 900 scenes: A combined source model of eye guidance. Visual Cognition, 17, 945-978. doi:10.1080/13506280902834720

FindLAY, J. M. (1997). Saccade target selection during visual search. Vision Research, 37, 617-631. doi:10.1016/S0042-6989(96)00218-0

Foulsham, T., \& UNDERWOOD, G. (2007). How does the purpose of inspection influence the potency of visual salience in scene perception? Perception, 36, 1123-1138.

Friedman, A. (1979). Framing pictures: The role of knowledge in automatized encoding and memory for gist. Journal of Experimental Psychology: General, 108, 316-355. doi:10.1037/0096-3445.108.3.316

Henderson, J. M. (1992). Object identification in context: The visual processing of natural scenes. Canadian Journal of Psychology, 46, 319-341. doi:10.1037/h0084325

HeNderson, J. M. (2003). Human gaze control during real-world scene perception. Trends in Cognitive Sciences, 7, 498-504. doi:10.1016/j tics.2003.09.006

Henderson, J. M., Pollatsek, A., \& Rayner, K. (1987). Effects of foveal priming and extrafoveal preview on object identification. Journal of Experimental Psychology: Human Perception \& Performance, 13, 449-463. doi:10.1037/0096-1523.13.3.449

Henderson, J. M., Weeks, P. A., JR., \& Hollingworth, A. (1999) The effects of semantic consistency on eye movements during complex scene viewing. Journal of Experimental Psychology: Human Perception \& Performance, 25, 210-228. doi:10.1037/0096 $-1523.25 .1 .210$

Hock, H. S., Gordon, G. P., \& Whitehurst, R. (1974). Contextual relations: The influence of familiarity, physical plausibility, and belongingness. Perception \& Psychophysics, 16, 4-8.

Hollingworth, A., \& Henderson, J. M. (1998). Does consistent scene context facilitate object perception? Journal of Experimental Psychology: General, 127, 398-415.

Kanan, C., Tong, M. H., Zhang, L., \& Cottrell, G. W. (2009). SUN Top-down saliency using natural statistics. Visual Cognition, 17, 9791003. doi: $10.1080 / 13506280902771138$

Loftus, G. R., \& MAcKworth, N. H. (1978). Cognitive determinants of fixation location during picture viewing. Journal of Experimental Psychology: Human Perception \& Performance, 4, 565-572. doi:10.1037/0096-1523.4.4.565

Malcolm, G. L., \& Henderson, J. M. (2009). The effects of target template specificity on visual search in real-world scenes: Evidence from eye movements. Journal of Vision, 9(11, Art. 8). doi:10.1167/9.11.8

McCarley, J. S., Kramer, A. F., Wickens, C. D., Vidoni, E. D., \& Boot, W. R. (2004). Visual skills in airport-security screening. Psychological Science, 15, 302-306. doi:10.1111/j.0956-7976.2004.00673.x

Neider, M. B., \& Zelinsky, G. J. (2006). Scene context guides eye movements during visual search. Vision Research, 46, 614-621. doi:10.1016/j.visres.2005.08.025

Pomplun, M., Ritter, H., \& Velichkovsky, B. (1996). Disambiguating complex visual information: Towards communication of personal views of a scene. Perception, 25, 931-948. doi:10.1068/p250931

Rayner, K., \& Pollatsek, A. (1992). Eye movements and scene perception. Canadian Journal of Psychology, 46, 342-376. doi:10.1037 h0084328

Rutishauser, U., \& Косн, C. (2007). Probabilistic modeling of eye movement data during conjunction search via feature-based attention Journal of Vision, 7(6, Art. 5). doi:10.1167/7.6.5

Schmidt, J., \& Zelinsky, G. J. (2009). Search guidance is proportional to the categorical specificity of a target cue. Quarterly Journal of Experimental Psychology, 62, 1904-1914. doi:10.1080/17470210902853530

Scialfa, C. T., \& Joffe, K. M. (1998). Response times and eye movements in feature and conjunction search as a function of target eccentricity. Perception \& Psychophysics, 60, 1067-1082.

Sheinberg, D. L., \& Logothetis, N. K. (2001). Noticing familiar objects in real world scenes: The role of temporal cortical neurons in natural vision. Journal of Neuroscience, 21, 1340-1350.

TATLER, B. W. (2009). Current understanding of eye guidance. Visual Cognition, 17, 777-789. doi:10.1080/13506280902869213

Torralba, A., Oliva, A., Castelhano, M. S., \& Henderson, J. M. (2006). Contextual guidance of eye movements and attention in realworld scenes: The role of global features in object search. Psychological Review, 113, 766-786. doi:10.1037/0033-295X.113.4.766

VickerY, T. J., King, L.-W., \& JiANG, Y. (2005). Setting up the target template in visual search. Journal of Vision, 5(1, Art. 8). doi: $10.1167 / 5.1 .8$

Võ, M. L.-H., \& Schneider, W. X. (2010). A glimpse is not a glimpse: Differential processing of flashed scene previews leads to differential target search benefits. Visual Cognition, 18, 171-200. doi:10.1080/13506280802547901

Williams, D. E., \& Reingold, E. M. (2001). Preattentive guidance of eye movements during triple conjunction search tasks: The effects of feature discriminability and saccadic amplitude. Psychonomic Bulletin \& Review, 8, 476-488.

Wolfe, J. M. (1998). Visual search. In H. Pashler (Ed.), Attention (pp. 13-73). Hove, U.K.: Psychology Press. 
Wolfe, J. M., Horowitz, T. S., Kenner, N., Hyle, M., \& Vasan, N. (2004). How fast can you change your mind? The speed of topdown guidance in visual search. Vision Research, 44, 1411-1426. doi:10.1016/j.visres.2003.11.024

Yarbus, A. L. (1967). Eye movements and vision. New York: Plenum.

ZelinsKy, G. J., \& SchmidT, J. (2009). An effect of referential scene constraint on search implies scene segmentation. Visual Cognition, 17, 1004-1028. doi:10.1080/13506280902764315

Zhang, L., Tong, M. H., \& CotTrell, G. W. (2007). Information attracts attention: A probabilistic account of the cross-race advantage in visual search. In D. S. McNamara \& J. G. Trafton (Eds.), Proceedings of the 29th Annual Conference of the Cognitive Science Society (pp. 749-754). Austin, TX: Cognitive Science Society.

Zhang, L., Tong, M. H., Marks, T. K., Shan, H., \& Cottrell, G. W. (2008). SUN: A Bayesian framework for saliency using natural statistics. Journal of Vision, 8(7, Art. 32). doi:10.1167/8.7.32

\section{NOTES}

1. Changes were completed by a computer graphics technician, and a separate group of 10 naive participants examined the scene to ensure that the location of the changes could not be identified during the scenepicture cue. None of the alterations to the photographs were identified by any of the participants.

2. A separate group of participants were shown the individual target items and were asked to identify them in order to ensure that the targetpicture cues were recognizable. The target objects included in the experiments were all correctly identified by the participants.

3. Heat maps were calculated using MATLAB code originally written by Aude Oliva.

(Manuscript received April 6, 2009;

revision accepted for publication March 6, 2010.) 